déprimé transversalement au milieu et, de chaque còté, un tubercule mousse. Cuisses assez renflées, tous les tibias épaissis au milieu et un peu arqués, les intermédiaires avec un fort éperon apical. ơ. - Long. 1,60 mill.

Cette espèce doit ètre placée dans la mème division que cimnamomeus Schfs. et tout près d'elle, mais elle en est très distincte par la forme de l'excavation céphalique; sous ce rapport, elle ressemblerait à honestus Schfs., mais chez cette dernière l'épistome porte une corne, tandis que cette partie de la tête est mutique chez Wasmanni, la corne antérieure appartenant au rebord du front et non à l'épistome.

Un seul exemplaire ơ de San Leolpoldo, Rio Grande del Sol (Brésil) [Heyer], avec Prinolepis fulva Mayr.

Je dois cet insecte à la générosité du Rèv. Père Wasmann.

\title{
Diagnoses préliminaires \\ d'espèces nouvelles de Sarcoptides plumicoles [ACAR.]
}

Par le Dr E. Trouessart.

\section{G. Ereyana s.-g nicrospalax.}

Freyana (Microspalax) delicatula, sp. n.

En ovale court, les flancs élargis, en arrière du sillon thoracique, par une lame mince; téguments minces et transparents. - Wäle à abdomen échancré angulairement, chaque lobe triangulaire portant sur son bord externe deux longs poils et un court; organe génital gros, bilobé, au niveau de la $3^{\text {e }}$ paire de pattes. - Femelle semblable au mìle mais à abdomen entier, arrondi, crénelé sur la ligne médiane où les poils abdominaux s'insèrent sur des lobules courts en chandelier; vulve dépourvue d'épimérite transversal. Épimères libres dans les deux sexes; plaque dorsale striée longitudinalement. — Long. totale: $0^{\mathrm{mm}}, 30$ a 32 (dans les deux sexes); larg. : $0^{\mathrm{mm}}, \mathbf{2 2}$ - Sur Dendrochelidon mystacina (Nouvelle-Guinée). C'est la première espèce de ce genre que l'on rencontre sur un Oiseau de l'ordre des Passereaux.

\section{G. IPterolichus s. $-g$. IProtolichus.}

\section{Pterolichus Protolichus) megamerus, sp. n.}

Mâle a pattes de la $\mathbb{I}^{\text {re }}$ paire près du double plus longues que celles de la 2 , avec les deux derniers articles renflés en forme de massue; corps de forme allongée; abdomen échancré, formant deux lobes trian- 
gulaires; lame de renforcement des lobes en Y renversé, circonscrivant l'organe génital. - Femelle plus courte, à pattes antérieures normales, ayant l'extrémité de l'abdomen bilobée et ornée de poils en feuille dentelés. - Long. total. : mâle $0^{\mathrm{mm}}, 37$ (patte antér. : $0^{\mathrm{mm}}, 30$ ); femelle $0^{\mathrm{mm}}, \ddot{0} 0$ - Sur Poiocephalus fuscicapillus (Afrique intertropicale).

\section{Pterolichus (Protolichus) chelidurus, sp. n.}

Male à abdomen prolongé en deux lobes divergents, tronqués à leur extrémité. a bord externe concave avec une échancrure triangulaire entre les deux, chaque lobe portant deux poils longs et un piquant, ce dernier inséré sur le bord externe. Organe génital circonscrit par les plaques de renforcement des lobes qui se rejoignent en forme de $\Lambda$. Glandes rouges bien visibles aux épimères et a la base des plaques de renforcement. Épimères antérieurs libres. - Long. totale : mảle $0^{\mathrm{mm}}, \breve{2}$; femelle $0^{\mathrm{mm}}, \mathbf{4 6}$. - Sur Bolborhynchus panychlorus (Guyane).

\section{Pterolichus (Protolichus) velifer, sp. n.}

(Pterolichus affinis, p.. Trt. et Mégn., Journ. Microg., 188., p. 262.)

Mâle à abdomen élargi à l'extrémité, fortement échancré en plein cintre, avec l'échancrure comblée par une lame mince, transparente, échancrée ou sinuée sur la ligne médiane; chaque lobe se termine en outre par une lame discoïdale, non plissée, insérée obliquement en dedans et dont le bord antéro-interne est soutenu par un renforcement en forme de piquant; un poil long et fort inséré, en dehors, avant l'extrémité du lobe. La plaque de renforcement qui soutient les lobes abdominaux se prolonge jusqu'au niveau de la $3^{\text {e }}$ paire de pattes qui est un peu moins forte que la $\mathbf{4}^{\mathrm{e}}$. Épimères antérieurs à extrémité libre. - Long. totale : mâle $0^{\mathrm{mm}}, 60$; femelle $0^{\mathrm{mm}}, \breve{0} 0$. - Sur Nymphicus cormutus (Nouvelle-Calédonie), Platycercus flaveolus (Australie), et Plat. personatus (ìles Fidji).

\section{Pterolichus Protolichus) Favettei, sp. n.}

Semblable au précédent, mais un peu plus allongé. Mâle à abdomen non élargi à l'extrémité, mais terminé par deux lobes allongés, triangulaires, aigus, fortement chitinisés, avec les poils abdominaux (deux grands et un plus petit, l'antérieur) rejetés sur le côté externe du lobe; sur le côté interne de chaque lobe, dans l'échancrure, un gros poil dilaté et tronqué en forme de hache; au fond de l'échancrure un petit lobe triangulaire forme saillie de chaque côté. Une grosse glande rouge a l'épimère de la $2^{\text {e }}$ paire de pattes. - Long. tot. : mâle $0^{\mathrm{mm}}, 68$; fe- 
melle $0^{\mathrm{mm}}, 60$. - Sur Nestor notabilis, Nestor meridionalis (NouvelleZélande), Psephotus xan thorrhoa (Australie) et Wicroglossum aterrimum (Nouvelle-Guinée). - Cette espèce est dédiée à M. le $\mathrm{D}^{\mathrm{r}}$ Favette, de Sain-Bel, dont les belles préparations et les microphotographies ont singulièrement facilité mes recherches sur les Sarcoptides plumicoles.

\section{S.-g. Roterolichus prop. dict.}

Pterolichus rubidus petaliferus, subsp. $\mathrm{n}$.

Semblable au type, mais les poils adbominaux, chez le mâle et la femelle, courts, égaux, étalés en forme de feuille ovale, lanceolée. - Sur Diomedea chlororhyncha (ìle Saint-Paul).

\section{Pterolichus umbellifer, sp. n.}

Oblong, l'abdomen entier dans les deux sexes; tout le pourtour du corps orné de poils en feuilles, dentelés ou digités a leur extrémité; les poils de l'épistome réduits à une seule paire, très grande, en forme de feuille ovale, plissée en éventail, dirigée en avant de manière à recouvrir le rostre dont elle dépasse l'extrémité. Deux paires de poils abdominaux simples. Les sexes ne diffèrent que par les organes génitaux. Plaque notogastrique largement criblée. - Long. tot. : $0^{\mathrm{mm}}, 30$. - Cette espèce rappelle Glyciphagus palmifer (Fumouze et Robin). Sur Opisthocomus hoazin, de la Guyane.

Pterolichus hystrix, sp. n.

Très semblable à l'espèce précédente, mais un peu plus court et plus large, tout le corps couvert de poils épineux, fasciculés. Les deux sexes ne diffèrent que par les organes génitaux. - Rappelle Glyciphagus plumiger (Fum. et Robin), et comme l'espèce précédente pourrait ètre prise, au premier abord, pour un Glyciphage. - Sur Opisthocomus hoazin, vivant en société avec le précédent.

\section{Pterolichus onychophorus, sp. n.}

Mâle à abdomen bilobé, échancré en plein cintre, chaque lobe se terminant par une lame mince, triangulaire, terminée par une pointe aiguë légèrement recourbée en dedans. Deux poils longs et un piquant, en dehors du lobe. Un piquant et un poil long sur les flancs. Pattes robustes. Branche libre des mandibules très forte, dépassant l'extrémité du rostre. - Femelle, à abdomen plus long, arrondi, avec une perforation ovale a la plaque notogastrique. — Long. tot. : màle, $0{ }^{\mathrm{mm}}, \breve{0} 0$ (avec les lames); femelle, $0^{\mathrm{mm}}, \breve{0} 0$. - Sur Brachypteracias Crossleyi Madagascar). 
Pterolichus onychophorus faunus, subsp. n.

Mile a lames triangulaires de l'abdomen rudimentaires ou nulles, l'abdomen étant simplement bilobé et chaque lobe portant deux poils longs et un piquant. Branche libre des mandibules fortement recourbée en dehors en forme de corne. Màle homéomorphe et femelle à mandibules normales. - Sur Brachypteracias pittoüdes et Br. Crossleyi avec le type (Madagascar).

\section{Pterolichus venustissimus, sp. n.}

Voisin de Pt. denticulatus mais plus petit, plus allongé, à formes délicates et téguments peu colorés; les dentelures des pattes et les poils en feuilles très développés; un poil en feuille rabattu en arrière au sillon thoracique, un autre lancéolé en avant de la $3^{\text {e }}$ paire de pattes. - Mâle à poils de l'échancrure abdominale dilatés en forme de feuille tronquée carrément et soutenue par une nervure oblique; les deux poils médians de chaque lobe allongés, lancéolés, en lame de cimeterre, à extrémité effilée. - Femelle à feuilles abdominales dilatées, ovales, finement dentelées. - Long. tot. : mile, $0^{\mathrm{mm}}, 37$; lemelle $0^{\mathrm{mm}}, \mathbf{4 0}$; larg. : $0^{\mathrm{mm}}, 10$. - Sur Conurus cunicularis (Mexique sud) et d'autres espèces de Conurus.

\section{Diagnose d'un nouvel Hydraena des Alpes françaises [CoL.]}

Par L. Bedel.

Hydraena heterogyna n. sp. - Oblonga, latiuscula, supra perparum convexa, nitida, picea, extremo margine elytrorum, paipis pedibusque rufis. Palpi in utroque sexu simplices. Pronotum hexagonum, subcordatum. Elytra ovata, intus recte punctato-striata striis quinque subintegris, stria sexta postice tantum discreta), extrinsecus dense punctata, lateribus late reflexo-marginata. - Long. 2 mill.

of Elytra apice conjunctim rotundata. Tibiae simplices, posticae dimidio inferiore pilis longissimis fimbriatae.

+ Elytra apice in spinam acutissimam producta, inter spinas profunde, fere semicirculariter emarginata.

Alpes-Maritimes : Saint-Martin-Vésubie, au fond d'un petit ruisseau sortant d'une source.

Cette espèce est remarquable par ses élytres ovales, largement en gouttière sur les còtés et surtout par leur extrémité, fortement épineuse et profondément échancrée chez la femelle. Elle a été trouvée récem- 


\section{$2 \mathrm{BHL}$ Biodiversity Heritage Library}

Trouessart, E.-L. 1898. "Diagnoses préliminaires d'espèces nouvelles de Sarcoptides plumicoles [Acar.]." Bulletin de la Société entomologique de France 1898, 289-292.

View This Item Online: https://www.biodiversitylibrary.org/item/38046

Permalink: https://www.biodiversitylibrary.org/partpdf/244919

\section{Holding Institution}

Smithsonian Libraries

\section{Sponsored by}

Smithsonian

\section{Copyright \& Reuse}

Copyright Status: NOT_IN_COPYRIGHT

This document was created from content at the Biodiversity Heritage Library, the world's largest open access digital library for biodiversity literature and archives. Visit BHL at https://www.biodiversitylibrary.org. 